\title{
Model for a macroscopically disordered conductor with an exactly linear high-field magnetoresistance
}

\author{
V. Guttal and D. Stroud \\ Department of Physics, The Ohio State University, Columbus, Ohio 43210-1106, USA
}

(Received 21 December 2004; published 17 May 2005)

\begin{abstract}
We calculate the effective resistivity of a macroscopically disordered two-dimensional conductor consisting of two components in a perpendicular magnetic field. When the two components have equal area fractions, we use a duality theorem to show that the magnetoresistance is nonsaturating and at high fields varies exactly linearly with the magnetic field. At other compositions, an effective-medium calculation leads to a saturating magnetoresistance. We briefly discuss possible connections between these results and magnetoresistance measurements on heavily disordered chalcogenide semiconductors.
\end{abstract}

DOI: 10.1103/PhysRevB.71.201304

PACS number(s): 75.47.De, 61.43.Hv, 72.15.Gd

The resistivity of most homogeneous materials (metals or semiconductors) increases quadratically with magnetic field $H$ at low fields, and generally saturates at sufficiently large $H .{ }^{1}$ Exceptions may occur for materials with Fermi surfaces allowing open orbits, or for compensated homogeneous semiconductors, where the resistivity may increase without saturation, usually proportional to $H^{2}$. 1,2 Under some special conditions, the magnetoresistance can be linear in magnetic field. ${ }^{3}$

Recently, a remarkably large transverse magnetoresistance (TMR) has been observed in the doped silver chalcogenides $\mathrm{Ag}_{2+\delta} \mathrm{Se}$ and $\mathrm{Ag}_{2+\delta} \mathrm{Te}^{4,5}$ In these materials, over the temperature range from 4 to $300 \mathrm{~K}$, the resistivity increases approximately linearly with $H$ up to fields, applied perpendicular to the direction of current flow, as high as $60 \mathrm{~T}$. Moreover, the TMR is especially large and most clearly linear at pressures where the Hall resistivity changes sign. ${ }^{6} \mathrm{Be}-$ cause of this linearity, these materials may be useful as magnetic field sensors even at megagauss fields.

But beyond the possible applications, the origin of the effect remains mysterious. According to conventional theories, such narrow gap semiconductors should have a saturating TMR. Furthermore, since these materials contain no magnetic moments, a spin-mediated mechanism seems unlikely.

There are presently two proposed explanations for this quasilinear TMR. The first is a quantum theory of magnetoresistance (MR). ${ }^{7}$ The second proposed mechanism ${ }^{8}$ is that this nonsaturating TMR arises from macroscopic sample inhomogeneities. Such inhomogeneities could produce large spatial fluctuations in the conductivity tensor and hence a large TMR, especially at large $H$. This explanation seems plausible because the chalcogenides probably have a granular microstructure ${ }^{6}$ and hence a spatially varying conductivity.

The effective conductivity of media, with a spatially varying conductivity $\sigma(\mathbf{x})$, has been studied since the time of Maxwell, but a relatively few studies have considered the magnetoresistance. ${ }^{9-18}$ For a three-dimensional (3D) medium, the TMR of an isotropic metal does indeed vary linearly in $H$, when a small volume fraction $p \ll 1$ of inclusions of a different carrier density is added. ${ }^{15}$ But the TMR gener- ally does not remain strictly linear at higher concentrations of p. If the inclusions are strictly insulating, then the TMR does remain asymptotically linear if the TMR is computed within the effective-medium approximation, ${ }^{19}$ but its exact behavior is not known even in this case. Recent experiments on homogeneous semiconductors containing a gold inhomogeneity ${ }^{20}$ show a hugely enhanced but not strictly linear room temperature geometrical TMR (i.e., arising from inhomogeneities); this so-called extraordinary magnetoresistance has been successfully modeled, using finite-element techniques. $^{21}$

The model of Ref. 8 also assumes a film with a spatially varying conductivity. The inhomogeneities are described by an impedance network; the tensor nature of the magnetoconductivity is included by making each network element a four-terminal impedance. Their numerical solution suggests that, for the network to have a nonsaturating TMR one needs (i) carriers of two different signs, and (ii) a suitably defined average mobility $\langle\mu\rangle \sim 0$. When solved numerically and averaged over many disorder realizations, their model does indeed give a nonsaturating, approximately linear TMR over a broad field range. Obviously, it would be useful to have exact analytical statements to compare with these numerical results.

In this Rapid Communication, we present an idealized model of a disordered semiconducting film in two dimensions. The model assumes a macroscopically inhomogeneous film, consisting of two different types of conducting regions, denoted $A$ and $B$, with areal fractions $p_{A}$ and $p_{B}=1-p_{A}$. In each region, the conductivity tensor is that of a Drude metal in a transverse magnetic field, but the density and the sign of charge carriers can be different in the two regions. We will show that, when $p_{A}=1 / 2$, and the charge carriers have opposite signs, the TMR is asymptotically exactly linear at sufficiently strong magnetic fields. Moreover, the linearity can extend down to quite low magnetic fields. The corresponding Hall coefficient $R_{H, e}$ is found to vanish. If $p_{A} \neq 1 / 2$, the effective resistivity tensor $\rho_{e}$ cannot be calculated exactly. An effective-medium approximation (EMA), which agrees with the exact result at $p_{A}=1 / 2$, predicts that the resistivity saturates for any $p_{A} \neq 1 / 2$, and that $R_{H, e}$ changes sign at $p_{A}=1 / 2$. All these results are in rough agreement with recent 
experiments $^{6}$ (which are, however, carried out for $3 \mathrm{D}$ samples; see below). If the carriers have the same sign, no exact statements are possible, even at $p_{A}=1 / 2$. But even in this case the EMA predicts a linear TMR precisely at $p_{A}=1 / 2$, though smaller than for carriers of opposite sign.

We first prove the exact linearity of the TMR at $p_{A}=1 / 2$ for carriers of opposite sign and opposite mobility, using a duality argument. We consider a two-dimensional (2D) conductor with a spatially varying conductivity tensor $\sigma(\mathbf{x})$, and denote the effective conductivity tensor by $\sigma_{\mathrm{e}} . \sigma_{e}$ is a $2 \times 2$ tensor defined by $\langle\mathbf{J}\rangle=\sigma_{e}\langle\mathbf{E}\rangle$, where $\mathbf{J}$ and $\mathbf{E}$ are the positiondependent current density and electric field, and $\langle\cdots\rangle$ denotes a spatial average in the limit of a large sample and suitable boundary conditions (as discussed, for example, in Ref. 15). $\sigma_{e}$ is the quantity that would be measured as the sample conductivity in an experiment. To calculate $\sigma_{e}$, we use a duality theorem, ${ }^{10}$ which states that

$$
\sigma_{e}[\sigma(\mathbf{x})] \sigma_{e}\left[\sigma^{-1}(\mathbf{x})\right]=I,
$$

where $I$ is the $2 \times 2$ unit matrix. Here $\sigma_{e}[\sigma(\mathbf{x})]$ denotes the effective conductivity tensor of a material whose local conductivity tensor is position-dependent and equal to $\sigma(\mathbf{x})$.

Thus, the product of $\sigma_{e}$ for the system of interest, and that of a hypothetical "dual composite" whose local conductivity tensor $\sigma_{\mathbf{d}}(\mathbf{x})$ is the local resistivity tensor of the original material, equals the unit tensor.

We now apply this theorem to the following special case. Let the two components each have a free-electron conductivity, but carriers of opposite signs. For the first component

$$
\begin{gathered}
\sigma_{A, x x}=\sigma_{A, y y}=\frac{\sigma_{A, 0}}{1+H^{2}}, \\
\sigma_{A, x y}=-\sigma_{A, y x}=\frac{\sigma_{A, 0} H}{1+H^{2}},
\end{gathered}
$$

where $\sigma_{A, 0}$ is the zero-field conductivity. The dimensionless magnetic field $H=\mu_{A} B / c$, where $\mu_{A}=e \tau_{A} / m_{A}$ is an effective mobility of carriers of type $A, m_{A}$ is their effective mass, $e>0$ is the electron charge magnitude, and $\tau_{A}$ a characteristic relaxation time. For the second component, we assume

$$
\begin{gathered}
\sigma_{B, x x}=\sigma_{B, y y}=\frac{\sigma_{B, 0}}{1+k^{2} H^{2}} . \\
\sigma_{B, x y}=-\sigma_{B, y x}=\frac{\sigma_{B, 0} k H}{1+k^{2} H^{2}},
\end{gathered}
$$

with the dimensionless constant $k=-1$ (i.e., the two types of charge carriers have opposite signs). We also introduce $\mu_{B}=k \mu_{A}$ as the effective mobility of type- $B$ carriers. Finally, we assume that the composite contains an areal fraction $p_{i}=1 / 2(i=A$ or $B)$ of each component, and that the geometry is symmetric. "Symmetric" means that, if the components $A$ and $B$ were interchanged, $\sigma_{e}$ of the film will remain the same in the thermodynamic limit. There are many geometries, both ordered (e.g., checkerboard) and random, which are symmetric by this definition. If we make the usual Drude assumption that $\sigma_{i, 0}=n_{i} e\left|\mu_{i}\right|(i=A, B)$, where $n_{i}$ is the num- ber density of carriers of type $i$, then Eqs. (2)-(5) imply (i) that there are equal areal fractions of positive and negative charge carriers (but not that the total numbers of positive and negative charge carriers are equal); and (ii) that the mobilities $\mu_{A}$ and $\mu_{B}$ are equal and opposite, so that $\langle\mu\rangle=\sum_{i=A, B} p_{i} \mu_{i}=0$.

Given these assumptions, the tensors $\sigma_{A}$ and $\sigma_{B}$ satisfy the remarkable relationship

$$
\sigma_{A}^{-1}=\frac{1+H^{2}}{\sigma_{0}^{2}} \sigma_{B},
$$

where $\sigma_{0}=\left(\sigma_{A, 0} \sigma_{B, 0}\right)^{1 / 2}$. Since we have an equal proportion of components $A$ and $B$, distributed in some symmetrical (and isotropic) fashion, the dual composite has a conductivity tensor

$$
\sigma_{d}(\mathbf{x})=\frac{1+H^{2}}{\sigma_{0}^{2}} \widetilde{\sigma}(\mathbf{x})
$$

where $\widetilde{\sigma}(\mathbf{x})$ means the conductivity of a composite in which the $A$ and $B$ components are interchanged. Since $\sigma_{d}$ is just a multiple of the original conductivity tensor $\sigma(\mathbf{x})$, but with $A$ and $B$ components interchanged, and since by the assumption of a symmetric composite $\sigma_{e}[\sigma(\mathbf{x})]=\sigma_{e}[\widetilde{\sigma}(\mathbf{x})]$, it follows that

$$
\sigma_{e}\left[\sigma_{d}(\mathbf{x})\right]=\frac{1+H^{2}}{\sigma_{0}^{2}} \sigma_{e}[\sigma(\mathbf{x})] .
$$

We now apply Eq. (1) to this model, with the result

$$
\frac{1+H^{2}}{\sigma_{0}^{2}} \sigma_{e}^{2}[\sigma(\mathbf{x})]=I
$$

A physically acceptable solution to Eq. (9) must have the diagonal elements of $\sigma_{e}$ equal and positive, and off-diagonal elements equal and opposite. It is readily shown algebraically that the only such solution is

$$
\sigma_{e}[\sigma(\mathbf{x})]=\frac{1}{\sqrt{1+H^{2}}} \sigma_{0} I .
$$

The corresponding resistivity tensor $\rho_{e}$ is

$$
\rho_{e}=\sigma_{0}^{-1} \sqrt{1+H^{2}} I
$$

The TMR is defined by the relation $\Delta \rho_{e, x x}(H)=\left[\rho_{e, x x}(H)\right.$ $\left.-\rho_{e, x x}(0)\right] / \rho_{e, x x}(0)$. For this model, $\Delta \rho_{e, x x}(H)=\sqrt{1+H^{2}}-1$ becomes linear in $H$ for large enough $H$, and the corresponding Hall coefficient $R_{H}=\rho_{x y}(H) / H=0$. Thus, this calculation appears to reproduce the numerical results of Ref. 8, but analytically.

Since the duality argument is not sufficient to determine $\sigma_{e}$ for $p_{A} \neq 1 / 2$, we have used the EMA for such concentrations. The EMA is a simple mean-field approximation in which the local electric fields and currents are calculated as if a given region is surrounded by a suitably averaged environment. For the present model the EMA becomes ${ }^{22}$ 


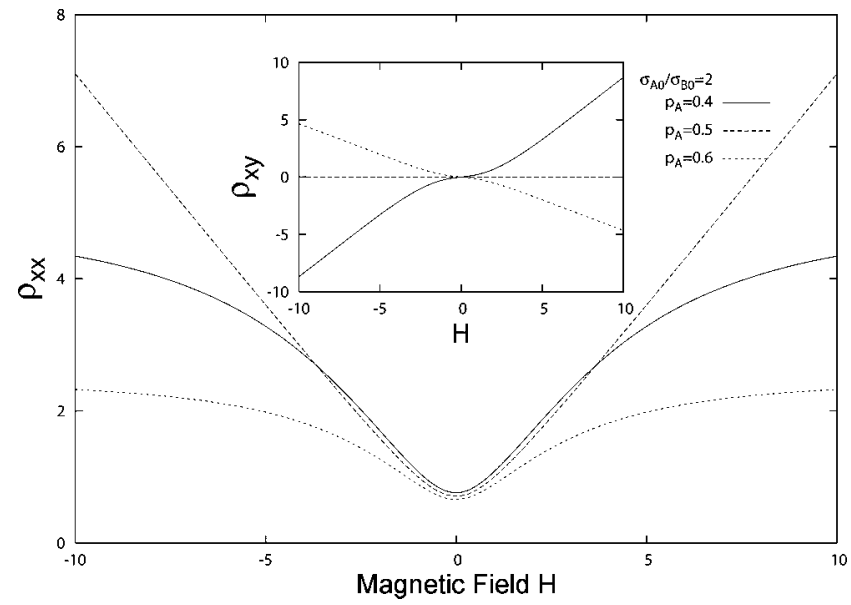

FIG. 1. Calculated transverse resistivity $\rho_{e, x x}\left(H, p_{A}\right)$ and Hall resistivity $\rho_{e, x y}\left(H, p_{A}\right)$ (inset) for a two-dimensional model inhomogeneous semiconductor in a transverse magnetic field, as calculated within the EMA for three different area fractions $p_{A}$ of component $A$. Both are given in units of $1 / \sigma_{0} \equiv 1 / \sqrt{\sigma_{A, 0} \sigma_{B, 0}}$. The two components $A$ and $B$ have conductivities given by Eqs. (2)-(5), with $\sigma_{A, 0} / \sigma_{B, 0}=2$. The mobilities of the two carriers are assumed to have the same magnitudes: $\left|\mu_{A}\right|=\left|\mu_{B}\right|$.

$$
\sum_{i=A, B} p_{i} \delta \sigma_{i}\left(I-\Gamma \delta \sigma_{i}\right)^{-1}=0
$$

Here $\delta \sigma_{i}=\sigma_{i}-\sigma_{e}$, and $\Gamma$ is a suitable depolarization tensor. We assume that $\sigma_{A}$ and $\sigma_{B}$ satisfy $\sigma_{i, x x}=\sigma_{i, y y} ; \sigma_{i, x y}=-\sigma_{i, y x}$. Then the components of $\sigma_{e}$ satisfy $\sigma_{e, x x}=\sigma_{e, y y}, \sigma_{e, x y}=-\sigma_{e, y x}$. We also assume that the two components $A$ and $B$ are distributed in compact, approximately circular regions. Then $\Gamma=-I /\left(2 \sigma_{e, x x}\right) .^{22}$ With these assumptions, Eq. (12) reduce to two coupled algebraic equations for $\sigma_{e, x x}$ and $\sigma_{e, x y}$ which are easily solved numerically.

To confirm that the EMA gives reasonable results, we have tested it for $p_{A}=p_{B}=\frac{1}{2}$, and $\sigma_{A}$ and $\sigma_{B}$ given by Eqs. (2) $-(5)$ with $k=-1$. We find that the solution to the Eq. (12) for the tensor $\sigma_{e}$ is diagonal, and a multiple of the unit tensor; the diagonal elements are given by Eq. (10). Thus, for $p_{A}=p_{B}$, the EMA agrees with the exact duality arguments.

To illustrate the EMA predictions for $p_{A} \neq \frac{1}{2}$, we calculate $\sigma_{e}$ for $\sigma_{i}$ given by Eqs. (2)-(5). The resulting elements of the resistivity tensor, $\rho_{e, x x}=\sigma_{e, x x} /\left[\sigma_{e, x x}^{2}+\sigma_{e, x y}^{2}\right], \rho_{e, x y}=$ $-\sigma_{e, x y} /\left[\sigma_{e, x x}^{2}+\sigma_{e, x y}^{2}\right]$, are plotted in Fig. 1 for $\sigma_{A 0} / \sigma_{B 0}=2$. Evidently, and as can be shown explicitly from the EMA equations, $\rho_{e, x x}$ is strictly linear in $H$ only at $p_{A}=1 / 2$. At all other concentrations, $\rho_{e, x x}(H)$ saturates (i.e., approaches a constant) at large $H$, but at a value much larger than $\rho_{e, x x}(H=0)$. It is easily shown that the saturation value of $\Delta \rho_{e, x x}\left(p_{A}\right) \equiv \operatorname{Lim}_{H \rightarrow \infty}\left[\rho_{e, x x}\left(H, p_{A}\right) / \rho_{e}\left(0, p_{A}\right)-1\right]$ $\propto 1 /\left|p_{A}-p_{c}\right|$ on both sides of the percolation threshold $p_{c}=1 / 2$. Figure 1 also shows that the effective Hall resistivity $\rho_{e, x y}$ changes sign just at the concentration where $\rho_{e, x x}$ varies asymptotically linearly with $H$.

We have also solved the EMA for a composite described by Eqs. (2)-(5) but for the more general case in which $k \neq-1$. Then $k>0$ and $k<0$ correspond, respectively, to car-

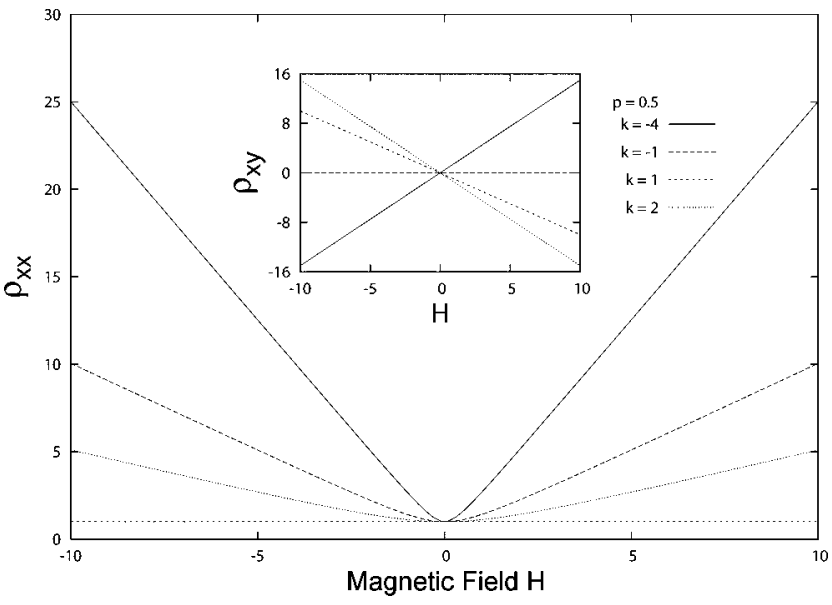

FIG. 2. Same as Fig. 1, but for $p_{A}=1 / 2$, and various choices of the mobility ratio $k=\mu_{B} / \mu_{A}$. A positive or negative $k$ means that the carriers have the same or opposite signs.

riers with mobilities of the same and opposite signs.

In Fig. 2 we show the EMA results for this model. Specifically, we show $\rho_{x x}\left(H, p_{A}\right)$ and $\rho_{x y}\left(H, p_{A}\right)$ with $p_{A}=1 / 2$, $\sigma_{A, 0}=\sigma_{B, 0}$, and several choices of $k$ corresponding to carriers of both opposite and the same sign. The case $k=1$ actually corresponds to a homogeneous free-electron metal. For all other values of $k$, the TMR is asymptotically linear, the linear behavior is evident even at moderate fields $(H \sim 1)$. However, the linear slope is larger when the carriers have opposite signs. We emphasize that these results are obtained in the EMA. The duality arguments do not give any predictions for $\rho_{x x}$ except when the carriers have opposite signs and opposite mobilities.

In Figs. 3 and 4, we plot the resistivity $\rho_{x x}$ and Hall coefficient $R_{H} \equiv \rho_{x y} / H$ as a function of $p_{A}$ for $H=1$ and $H=10$. In both cases, we assume that $\sigma_{A, 0}=\sigma_{B, 0}$ and $\left|\mu_{A}\right|=\left|\mu_{B}\right| \cdot \rho_{x x}$ has a peak at $p_{A}=1 / 2$, which sharpens, as a function of $p_{A}$, as $H$ increases. Similarly, the Hall coefficient $R_{H}$ changes sign at $p_{A}=1 / 2$, and the change occurs over a narrower and narrower regime of $p_{A}$ as $H$ increases.

The present results agree qualitatively with the experiments of Lee et al., ${ }^{6}$ which also show that the TMR peaks at pressures where the Hall coefficient changes sign. But this agreement should be viewed cautiously. In particular, the measurements of Ref. 6 are carried out on a 3D sample, while our calculations are for a 2D system. The present work would also apply to a 3D system with a columnar microstructure - that is, a system in which the conductivity tensor $\sigma(\mathbf{x})$ is independent of the third dimension, $z-$ and the applied field $\mathbf{B} \| z$, but the samples of Ref. 6 if inhomogeneous, are most likely composed of small compact grains. We have calculated $\sigma_{e}$ for a 3D granular sample with carriers of opposite signs, using the EMA, and find results similar to those shown here for $2 \mathrm{D}$ samples. These $3 \mathrm{D}$ calculations will be presented elsewhere. ${ }^{23}$

The TMR of the present model is very large$\Delta \rho_{x x}(H, 1 / 2) \sim 10$ for $H \sim 10$ - and remains approximately linear down to fields as low as $H \sim 1-2$. By contrast, other models of TMR that arises from inhomogeneities produce only a small TMR, or, if a large TMR, $\Delta \rho_{x x}(H)$ does not vary linearly with $H .{ }^{15,16}$ 


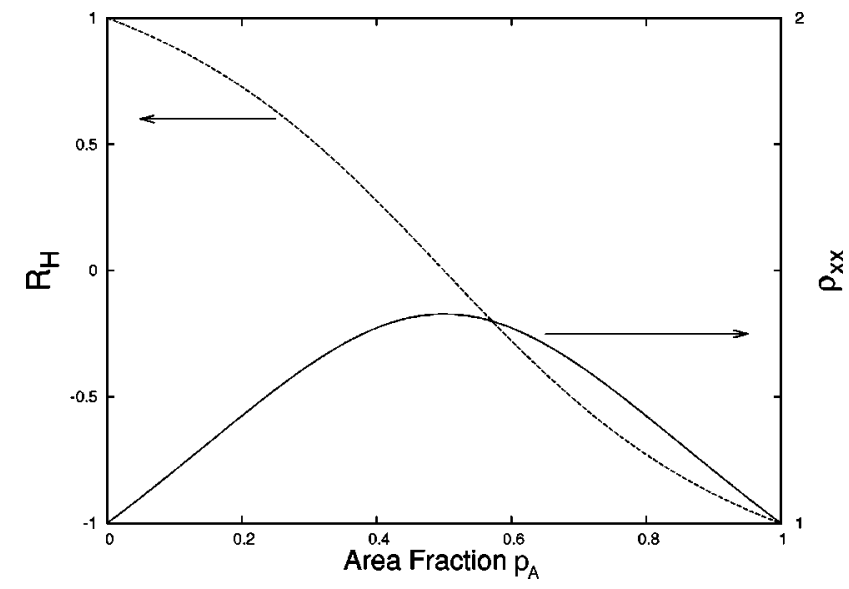

FIG. 3. Transverse resistivity $\rho_{x x}\left(H, p_{A}\right)$ and Hall coefficient $R_{H}\left(H, p_{A}\right)$ as a function of $p_{A}$ for $H=1$, using the same model as in Fig. 1, with $\sigma_{B, 0}=\sigma_{A, 0}$ and $\mu_{B}=-\mu_{A}$.

In summary, we have presented a simple model of a $2 \mathrm{D}$ macroscopically inhomogeneous material, whose TMR is asymptotically linear in magnetic field, and whose corresponding Hall coefficient vanishes. The model has several unusual properties that make it likely to be realized only in special circumstances. First, Eqs. (2)-(5) imply that the carriers have equal and opposite mobilities $\mu_{A}=-\mu_{B}$. Secondly, the linearity occurs only if the composite has a symmetric geometry at

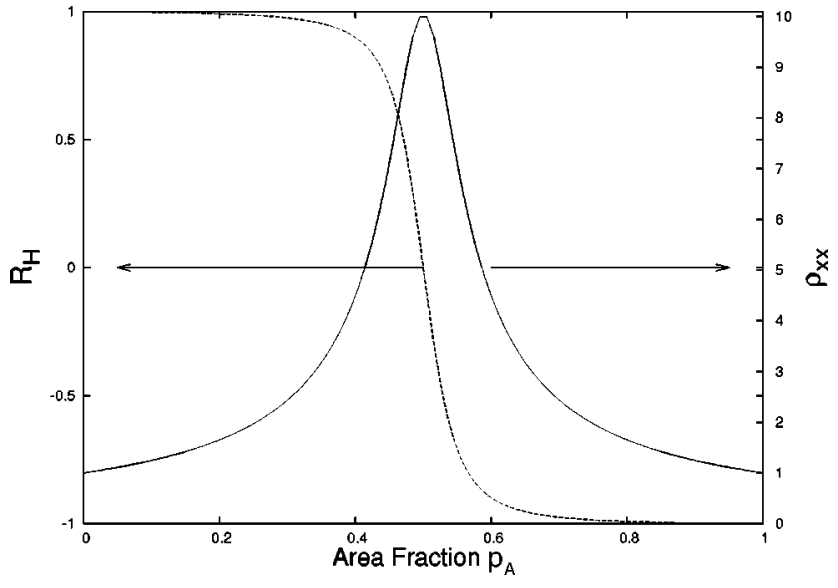

FIG. 4. Same as Fig. 3, but for $H=10$.

$p_{A}=1 / 2$. But given these features, the TMR, arising from a perpendicular to the sample, is asymptotically exactly linear in $B$. This is an analytically soluble model for TMR due to macroscopic inhomogeneities, which produces a linear TMR at high concentrations of inhomogeneities.

This work was supported by the National Science Foundation, through Grant No. DMR04-13395. We also benefited from the facilities of the Ohio Supercomputer Center.
${ }^{1}$ For a discussion, see, e. g., N. W. Ashcroft and N. D. Mermin, Solid State Physics (Saunders, Fort Worth, 1975), Chap. 12.

${ }^{2}$ See, e. g., J. M. Ziman, Principles of the Theory of Solids (Cambridge University Press, Cambridge, 1972), pp. 250-254.

${ }^{3}$ For a recent short review, see, e. g., A. A. Abrikosov, Europhys. Lett. 49, 789 (2000), and references therein.

${ }^{4}$ R. Xu, A. Husmann, T. F. Rosenbaum, M. L. Saboungi, J. E. Enderby, and P. B. Littlewood, Nature (London) 290, 57 (1997).

${ }^{5}$ A. Husmann, J. B. Betts, G. S. Boebinger, A. Migliori, T. F. Rosenbaum, and M. L. Saboungi, Nature (London) 417, 421 (2002).

${ }^{6}$ M. Lee, T. F. Rosenbaum, M.-L. Saboungi, and H. S. Schnyders, Phys. Rev. Lett. 88, 066602 (2002).

${ }^{7}$ A. A. Abrikosov, Phys. Rev. B 58, 2788 (1998).

${ }^{8}$ M. M. Parish and P. B. Littlewood, Nature (London) 426, 162 (2003).

${ }^{9}$ C. Herring, J. Appl. Phys. 31, 1939 (1960).

${ }^{10}$ K. S. Mendelson, J. Appl. Phys. 46, 4740 (1975).

${ }^{11}$ D. Stroud and D. J. Bergman, Phys. Rev. B 30, 447 (1984).
${ }^{12}$ G. J. C. L. Bruls, J. Bass, A. P. van Gelder, H. van Kempen, and P. Wyder, Phys. Rev. Lett. 46, 553 (1981).

${ }^{13}$ Yu. A. Dreizin and A. M. Dykhne, Sov. Phys. JETP 36, 127 (1973).

${ }^{14}$ Yakov M. Strelniker and D. J. Bergman, Phys. Rev. B 67, 184416 (2003), and references cited therein.

${ }^{15}$ D. Stroud and F. P. Pan, Phys. Rev. B 13, 1434 (1976).

${ }^{16}$ D. Stroud and F. P. Pan, Phys. Rev. B 20, 455 (1979).

${ }^{17}$ B. Ya. Balagurov, Sov. Phys. Solid State 28, 1694 (1986).

${ }^{18}$ A. K. Sarychev, D. J. Bergman, and Y. M. Strelniker, Phys. Rev. B 48, 3145 (1993).

${ }^{19}$ D. J. Bergman and D. G. Stroud, Phys. Rev. B 62, 6603 (2000).

${ }^{20} \mathrm{~S}$. A. Solin, T. Thio, D. R. Hines, and J. J. Heremans, Science 289, 1530 (2000).

${ }^{21}$ J. Moussa, L. R. Ram-Mohan, J. Sullivan, T. Zhou, D. R. Hines, and S. A. Solin, Phys. Rev. B 64, 184410 (2001).

${ }^{22}$ D. Stroud, Phys. Rev. B 12, 3368 (1975).

${ }^{23}$ V. Guttal and D. Stroud (unpublished). 